\title{
Análisis de cambios de la cobertura vegetal en la macrocuenca del Pacífico 2000-2009
}

\section{Analysis of changes in macrocuenca vegetation covers Pacific 2000-2009}

\section{Erika Palacios Bermúdez*}

\section{Resumen}

En los últimos años las coberturas vegetales de la macrocuenca del Pacífico han experimentado cambios significativos debido a las indiscriminadas y preexistentes intervenciones antrópicas en los ecosistemas, originando así importantes cambios en la disponibilidad de los recursos naturales existentes en los diversos hábitats. Considerando la situación anterior se realizó un análisis multitemporal de los cambios de las coberturas vegetales en la macrocuenca del Pacífico en un período de 9 años comprendido entre 2000 y 2009. En el análisis multitemporal se utilizaron imágenes de satélite del programa Landsat TMy ETM+, las cuales fueron interpretadas visualmente en pantallas de monitores con base en composiciones de colores prediseñada, falso color $R G B$ 4, 5, 3 (rojo, gris, azul). Las imágenes se procesaron utilizando el software (Erdas 9.2) lo cual permitió generar archivos en formato (.img) y transformarlos a vectoriales o polígonos distintivos, que se clasificaron de acuerdo con la metodología de clasificación de coberturas Corine Land Cober a escala 1:100.000. En el análisis de los datos obtenidos se utilizaron las cadenas de Markov, que permitieron determinar las áreas de las coberturas que cambiaron y las que permanecieron estables en un período determinado. La cobertura boscosa de la macrocuenca del Pacífico durante el período analizado presentó una reducción en su superficie de 173.718,30 hectáreas correspondiente a 3,3\% de la superficie registrada inicialmente en el año 2000

Palabras clave: Análisis multitemporal, Coberturas vegetales, Imágenes de satélite.

\begin{abstract}
In recent years the vegetation cover of the Pacific macrocuenca have undergone significant changes due to indiscriminate and existing anthropic interventions in ecosystems, thus causing major changes in the availability of natural resources in different habitats. Considering the above situation a multi-temporal analysis of changes in vegetation cover in the Pacific macrocuenca was conducted over a period of nine years from 2000 to 2009. In the multitemporal analysis of satellite images Landsat TM and ETM + program were used, the which they were interpreted visually monitor screens compositions based on pre-designed color, false color, $R G B$ 4, 5, 3 (red, gray, blue). The images were processed using the (Erdas 9.2) software which allowed generate format files (.img) and transform them to vector or distinctive polygons, which were classified according to the methodology cover classification Corine Land Cober at 1:100,000. In the analysis of data obtained Markov chains, which allowed us to determine coverage areas that changed and those that remained stable in a given period were used. The
\end{abstract}

* Ingeniera Teleinformática, estudiante Especialización en Sistemas de Información Geográfica, Instituto de Investigaciones Ambientales del Pacífico (IIAP). e-mail: epalacios@iiap.org.co

Recibido: 14 de noviembre de 2012

Aceptado: 28 de marzo de 2013 


\section{Bioetnia Volumen 10, 2013}

forest cover of the macrocuenca Pacific during the period under review showed a reduction in its area of 173,718.30 hectares corresponding to $3.3 \%$ of the initially registered in 2000 surface.

Keywords: Multitemporal analysis, Mulches, Satellite images.

\section{Introducción}

El análisis de las coberturas terrestres y el uso de la tierra representan una de las estrategias más importantes en el momento de adelantar estudios de esta naturaleza, considerando que a partir de estos ejercicios de investigación se puede establecer el estado que en un determinado tiempo presentan los recursos naturales y el grado de intervención que sobre ellos ejerce la población siempre y cuando se utilicen métodos adecuados para el levantamiento de la información. Por eso la teledetección se constituye en una poderosa herramienta para evaluar los cambios en diversas variables relacionadas con el funcionamiento ecosistémico (Alcaraz et al. 2008).

El uso que el hombre hace de los diferentes recursos naturales constituye una de las principales causas de deterioro ambiental donde sobresalen aspectos relacionados con la deforestación y fragmentación de los ecosistemas, la alteración de los ciclos hídricos, la degradación de los suelos y el incremento de la vulnerabilidad de los grupos humanos circundantes, tanto así, que la actividad humana ha sido la gran transformadora del paisaje en los últimos 300 años (Lambin 1997 citado por Ruiz et al. 2013).

En diversos estudios se afirma que la mitad del territorio colombiano está cubierto por bosques y que el país ocupa el tercer lugar en Suramérica después de Brasil y Perú, y es el quinto en la región respecto a la cobertura de bosque primario (bosques con alto grado de biodiversidad que nunca han sido explotados, fragmentados, o influidos directa o indirectamente por el hombre), con 8.5 millones de hectáreas; el resto, 51.5 millones de hectáreas, son de bosque regenerado y 350.000 hectáreas de plantaciones forestales [Organización de Naciones Unidas para la Agricultura y la Alimentación (FAO) 2010].

La región del Pacífico colombiano contiene $8 \%$ de los bosques naturales del país y la mitad de su territorio está cubierta por bosques, sobre todo selvas [Instituto Geográfico Agustín Codazzi (IGAC) et al. 2002]. En 1990 la cobertura boscosa en el país era de 64'442,269 hectáreas, es decir, $56.5 \%$ del territorio nacional; en el año 2010 la superficie de cobertura boscosa total descendió a 59'021,810 hectáreas, lo que indica que un período de 20 años se perdieron 5.4 millones de hectáreas de bosque, un área del tamaño de Costa Rica [Instituto de Hidrología, Meteorología y
Estudios Ambientales (IDEAM) 2011].

La compresión de la dinámica de la pérdida de cobertura a través del tiempo se hace mediante la realización de ejercicios de investigación como el que se describe en este capítulo, utilizando tecnologías de vanguardia que permitan generar información de calidad para la toma de decisiones adecuadas y pertinentes en la planificación, ordenación, manejo y aprovechamiento de los recursos naturales.

\section{Metodología}

Área de estudio. El estudio se realizó en el área de influencia de la macrocuenca del Pacífico, república de Colombia, Sur América, la cual ostenta una superficie de 7'727.376.58 hectáreas, correspondiente a $68.4 \%$ de la Reserva Forestal del Pacífico creada mediante Ley Segunda del año 1959. Se ubica al noroccidente de Colombia, con jurisdicción en los departamentos de Chocó, Risaralda, Valle del Cauca, Cauca y Nariño, extendiéndose desde el extremo norte del municipio de Juradó, Chocó hasta el extremo sur del municipio de Tumaco, Nariño, entre las coordenadas de los puntos extremos (Tabla 1), registrando una longitud de $740 \mathrm{~km}$ que corresponde a $40 \%$ de la longitud total de Colombia (Figura 1).

Método. Se realizó una revisión selectiva de información secundaria de proyectos con intereses particulares en el tema estudiado, que permitió seleccionar dos períodos de análisis de cambios de las coberturas vegetales 2000-2002 y 2005-2009. Se utilizaron imágenes de satélite generadas por el programa Landsat TM y ETM las cuales tienen mejores condiciones técnicas: 8 bandas, las dos últimas pancromática y multiespectral con resolución de 15 y $30 \mathrm{~m}$ respectivamente además que permiten una fácil identificación y clasificación de coberturas, vegetación y humedad. La información espacial se interpretó visualmente en pantallas de monitores para cada uno de los períodos evaluados con base en composiciones de color previamente elaboradas, mediante la combinación de falso color RGB 4,5,3 (rojo, gris, azul) y utilizando a la metodología Corine Land Cober a escala 1:100.000 en los períodos estudiados. Las imágenes

\section{Tabla 1}

Coordenadas de ubicación de puntos extremos de la macrocuenca del Pacífico

\begin{tabular}{cll}
\hline Ítem & Norte & Oeste \\
\hline 1 & $7^{\circ} 29^{\prime} 5.16^{\prime \prime}$ & $77^{\circ} 43^{\prime} 24.31^{\prime \prime}$ \\
2 & $0^{\circ} 43^{\prime} 45.08^{\prime \prime}$ & $77^{\circ} 33^{\prime} 55.89^{\prime \prime}$ \\
3 & $5^{\circ} 28^{\prime} 21.03^{\prime \prime}$ & $7^{\circ} 51^{\prime} 46.82^{\prime \prime}$ \\
4 & $1^{\circ} 36^{\prime} 12.69^{\prime \prime}$ & $78^{\circ} 59^{\prime} 27.82^{\prime \prime}$ \\
\hline
\end{tabular}




\section{Cambios de la cobertura vegetal. E Palacios}

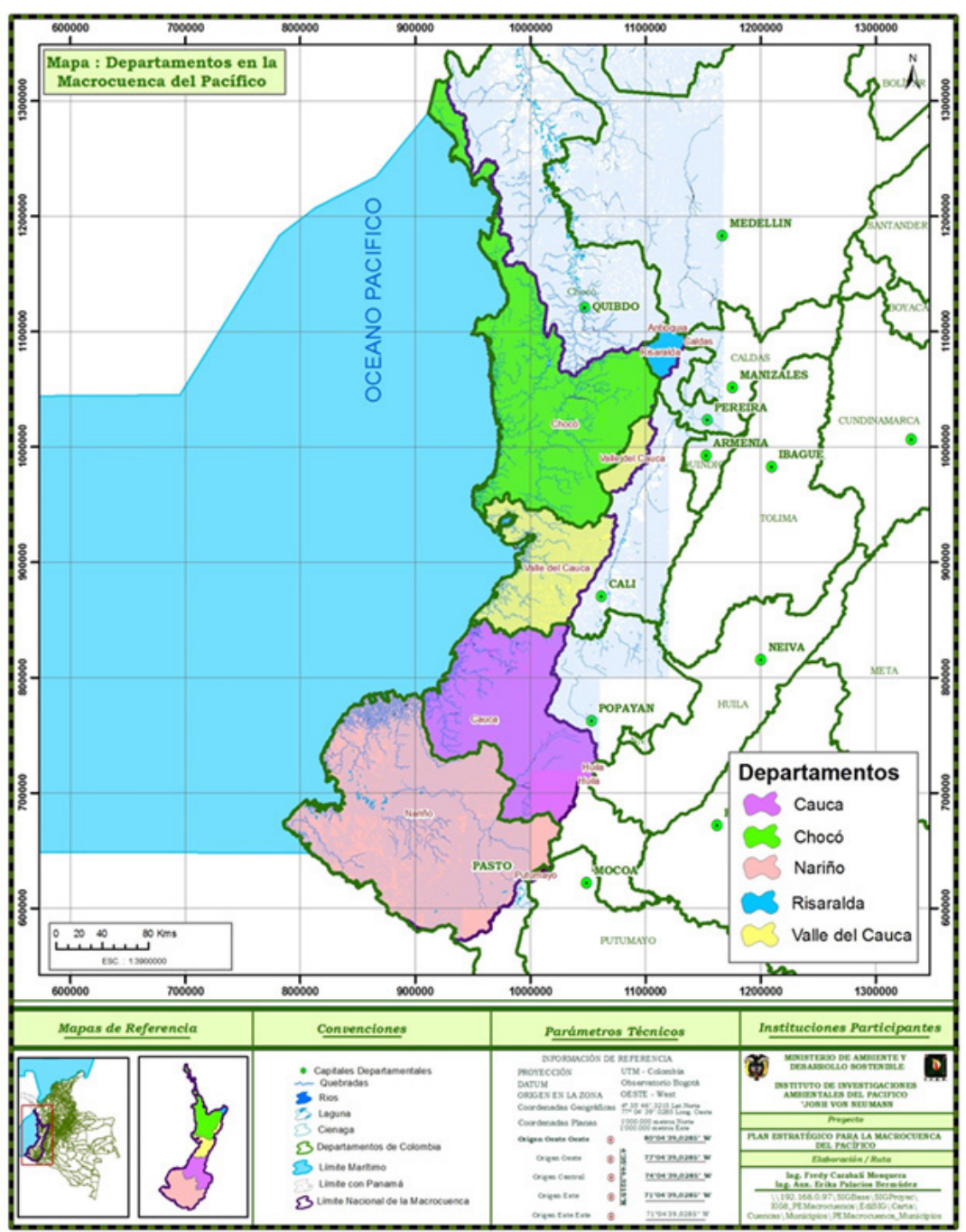

Figura 1. Mapa de ubicación de la macrocuenca del Pacífico.

de satélite se procesaron utilizando el software (Erdas 9.2) lo cual permitió generar archivos en formato .img y transformarlos a vectoriales o polígonos distintivos de cada una de las coberturas interpretadas en las imágenes de satélite, que alimentaron las bases de datos. El cálculo del valor de las superficies de las coberturas se realizó utilizando los archivos en formato Shapefile (vectoriales) y se procesaron con el software ArcGIS 10.2. Para analizar las variables ocultas en los cambios temporales de las coberturas se utilizó las cadenas de Márkov ${ }^{1}$ (Figura 2) y se interpretaron con

\footnotetext{
En la teoría de la probabilidad, se conoce como cadena de Márkov o modelo de Márkov a un tipo especial de proceso estocástico discreto en el que la probabilidad de que ocurra un evento depende solamente del evento inmediatamente anterior. Esta característica de falta de memoria recibe el nombre de propiedad de Markov.
}

base en la metodología Corine Land Cober.

El análisis multitemporal se realizó a través de la comparación de los mapas de clasificación por categorías de coberturas terrestres mediante la superposición temática, la cual permitió detectar los cambios de cobertura mediante la combinación de la información de categorías con una tabulación cruzada de las fechas consideradas.

\section{Resultados y discusión}

Se cuantificaron las coberturas de uso del suelo de la macrocuenca del Pacífico para los períodos 2000-2002 y 2005-2009, delimitada en una superficie de 7.703.940 hectáreas y representadas en 13 subtipos de coberturas (Tabla 2) según la metodología Corine Land Cober. Se registró una cobertura adicional denominada "sin información" no descrita en la metodología mencionada, con superficies de 


\section{Bioetnia Volumen 10, 2013}

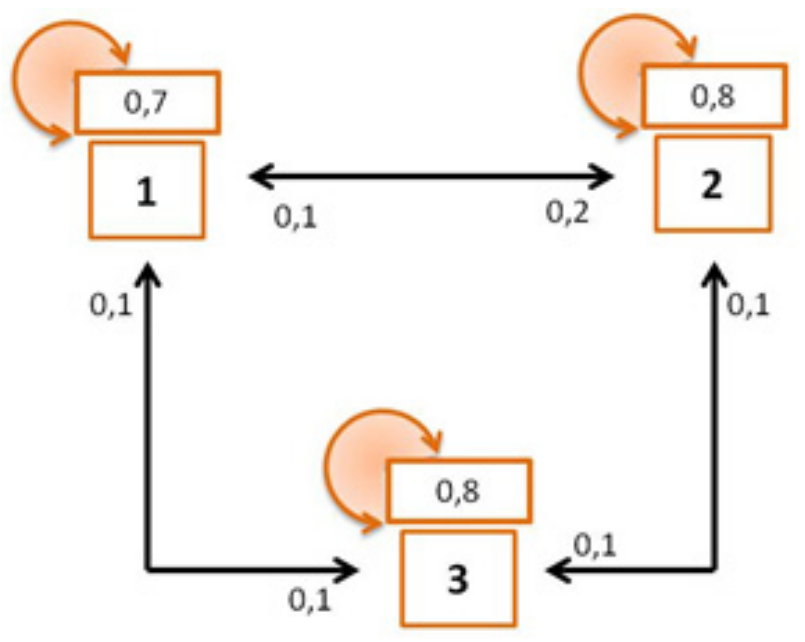

Figura 2. Cadena de Markov en un sistema de coberturas terrestres.

443.260 y 64.337 hectáreas en cada uno de los períodos estudiados respectivamente. La aparición de superficies $\sin$ información obedece a que la zona de estudio permanece bajo influencia de nubosidad durante gran parte del año (IDEAM et al. 2010) pero también a problemas del sensor (bandeamiento de imágenes Landsat).

Durante el período 2005-2009 se redujo en 3.3\% de la cobertura vegetal correspondiente a los bosques naturales. Lo cual está relacionado en parte con el aumento de las superficies de cultivos ilícitos de coca en 25.167 hectáreas en los departamentos de Nariño, Cauca, Chocó, y Valle del Cauca [Organización de Naciones Unidas contra la Droga y el Delito (UNODC) 2010]. Por su parte el Departamento Nacional de Planeación (DPN 2007) atribuye los procesos de deforestación a la expansión de la frontera agropecuaria y la colonización, incluidos los cultivos ilícitos, los incendios forestales y la extracción de madera. En el departamento del Chocó durante el año 2009 se extrajeron $222.939 \mathrm{~m}^{3}$ lo cual soporta en parte la pérdida de cobertura de bosques producto de la extracción selectiva [Corporación Autónoma Regional para el Desarrollo Sostenible del Chocó (CODECHOCÓ) 2015].

La dinámica de cambio de la cobertura de los bosques, hacia los demás "grandes grupos de coberturas" que según la metodología Corine Land Cober conforman el paisaje, se registró una tendencia de cambio de $0.008 \%$ hacia territorios artificializados, $8.3 \%$ a territorios agrícolas, $2.5 \%$ a áreas seminaturales y $0.5 \%$ a áreas húmedas y superficies de agua según las matrices de probabilidades de cambio de las cadenas de Markov (Tabla 3).

Entre los períodos evaluados se cuantificó un aumento en la superficie de áreas agrícolas heterogéneas en 35.6\%,

Tabla 2

Áreas de coberturas de uso del suelo en la macrocuenca del Pacífico

\begin{tabular}{|c|c|c|c|}
\hline \multirow[t]{2}{*}{ Código } & \multirow[t]{2}{*}{ Cobertura } & \multicolumn{2}{|c|}{ Área } \\
\hline & & $2000-2002$ & $2005-2009$ \\
\hline 0 & Sin información* & 443.260 & 64.337 \\
\hline 11 & Zonas urbanizadas & 3.337 & 4.127 \\
\hline 12 & Zonas industriales o comerciales y redes de comunicación & 497 & 502 \\
\hline 13 & Zonas de extracción minera y escombreras & 781 & 1.103 \\
\hline 21 & Cultivos transitorios & 6.277 & 4.446 \\
\hline 22 & Cultivos permanentes & 90.383 & 92.913 \\
\hline 23 & Pastos & 248.463 & 232.321 \\
\hline 24 & Áreas agrícolas heterogéneas & 960.567 & $1 ’ 490.751$ \\
\hline 31 & Bosques & $5 ’ 342.188$ & $5 ’ 168.469$ \\
\hline 32 & Áreas con vegetación herbácea y/o arbustiva & 464.182 & 488.820 \\
\hline 33 & Áreas abiertas, sin o con poca vegetación & 3.328 & 2.216 \\
\hline 41 & Áreas húmedas continentales & 8.329 & 13.539 \\
\hline 42 & Áreas húmedas costeras & 1.975 & 1.427 \\
\hline \multirow[t]{2}{*}{51} & Aguas continentales & 130.373 & 138.969 \\
\hline & Total & 7703.938 & 7'703.938 \\
\hline
\end{tabular}

* Cobertura no descrita en la metodología Corine Lan Cober. 
Cambios de la cobertura vegetal. E Palacios

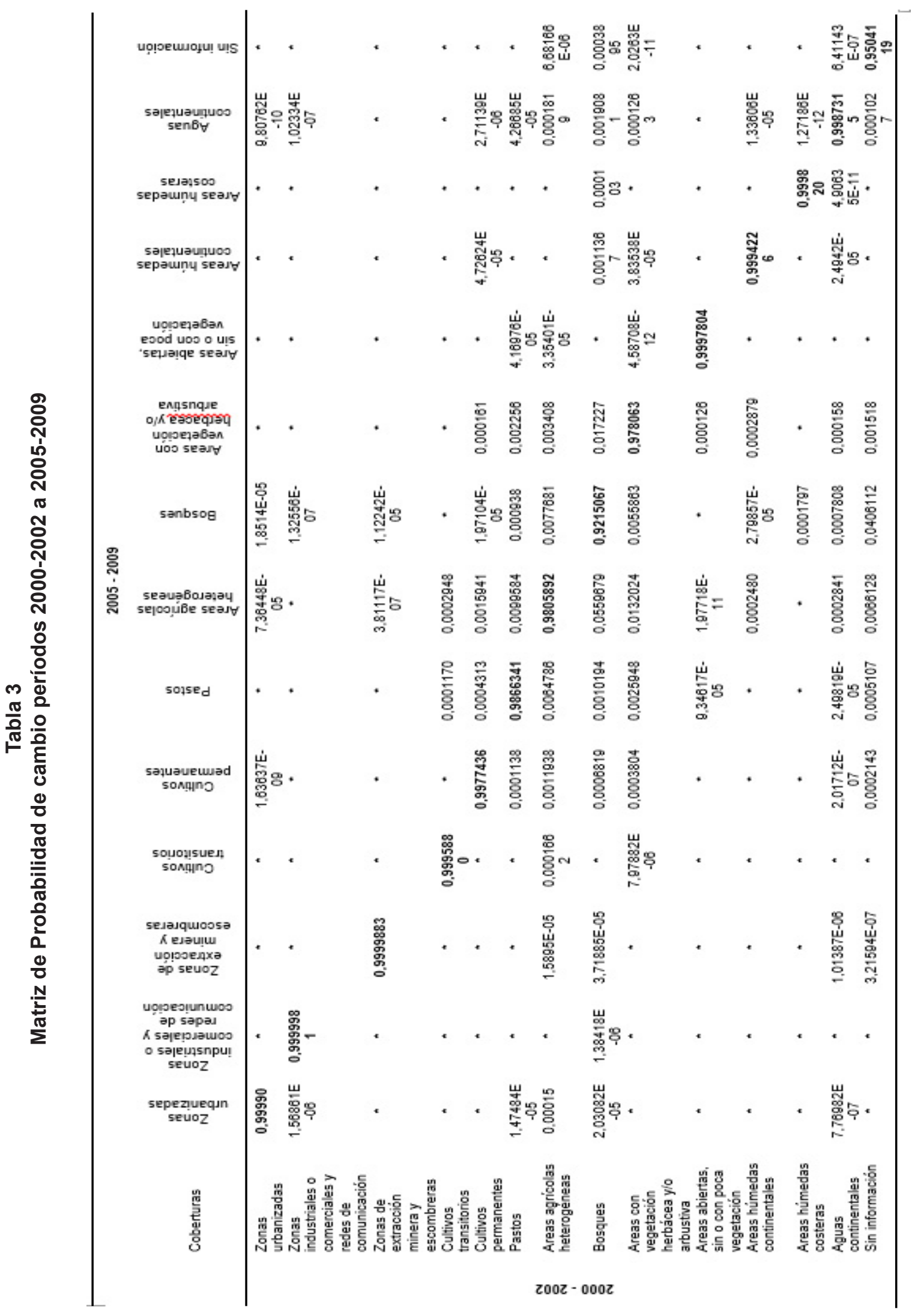




\section{Bioetnia Volumen 10, 2013}

debido principalmente a la expansión hacia áreas de cobertura de bosques y los territorios agrícolas, producto de la fragmentación del paisaje natural y de la tendencia de los productores a combinar actividades agrícolas con actividades ganaderas.

La cobertura vegetación herbáceay/o arbustiva aumentó su superficie en 5\%, lo cual se atribuye al aprovechamiento selectivo que se adelantó en algunas zonas boscosas donde se extrajeron volúmenes de madera superior a $2.000 \mathrm{~m}^{3}$ de algunas especies de alto valor comercial como abarco (Cariniana pyriphormis), algarrobo (Hymenea palustre), bálsamo (Miroxylum balsamum), caimito (Chrysophyllum auratm), carrá (Huberodendrum patinoi), cedro (Cedrela odorata), chano (Sacoglotis procera), guasca (Eschuwler sp.), guino (Carapa guianensis), lechero (Brosimun utile), lirio(Couma macrocarpa), machare (Hymenaea ebloungifolia), nuanamo(Virola sp.), Otobo(Dialyantheragracilipes), sajo (Campnosperma panamensis) (CODECHOCO 2015) y por el abandono de áreas agrícolas y ganaderas (influidas por cambios voluntarios en los sistemas de producción de las poblaciones asentadas en la macrocuenca y por presiones de grupo armados dando lugar a migraciones y desplazamientos (UNODC 2010) permitiendo así la recuperación natural de estas áreas.

Las coberturas aguas continentales y áreas húmedas continentales presentan un aumento considerable del 10\%, situación que de mantenerse en el futuro, permitirá generar grandes expectativas respecto a la disponibilidad de este importante recurso en beneficio de las comunidades, mejorando sus condiciones de vida y las posibilidades de un mejor desarrollo económico y social para la región. La mayor intervención de las coberturas de uso del suelo se desarrolló en la región suroccidental de la macrocuenca del Pacífico (Figura 3). Las demás clases de coberturas que complementan el paisaje presentan una aparente estabilidad para este período (Tabla 4).

\section{Conclusiones}

Los bosques de la macrocuenca del Pacífico durante los períodos 2000-2002 y 2005-2009 presentaron una reducción en su superficie de 173.718,30 hectáreas correspondiente al 3,3\% cifra no significativa en relación con la superficie inicial registrada en el año 2000; la degradación de esta cobertura obedece sobre todo a procesos de intervención antrópica como la expansión de las fronteras agrícolas, el desarrollo de actividades mineras, las siembra de cultivos ilícitos y de palma de aceite africana desarrollados principalmente en la región sur y centro.

La mayor parte de las coberturas de uso del suelo descritas en la macrocuenca del Pacífico a excepción de la cobertura de bosques desarrollada sobre todo en la región

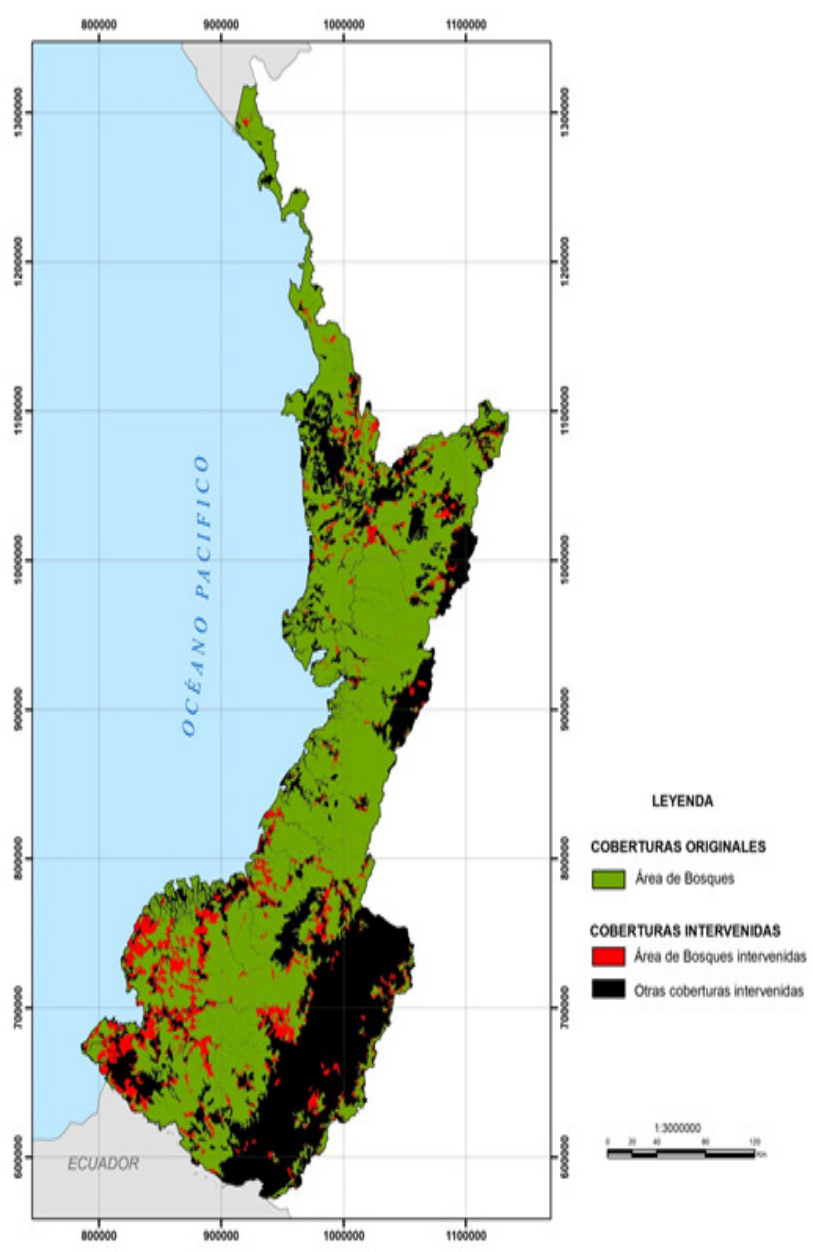

Figura 3. Mapa transición de cambios de cobertura en la macrocuenca del Pacífico períodos 2000-2002 y 2005-2009.

suroriental y centro-oriente presentan un desarrollo normal de acuerdo con las dinámicas de cambio de las coberturas del suelo. Este estudio permitió evidenciar que la tendencia de uso del suelo orientada a generar procesos de producción más sostenibles pasando de monocultivos a cultivos heterogéneos, haciendo aprovechamientos forestales permanentes y permitiendo que los procesos de regeneración natural se completen.

Los procesos de expansión de zonas urbanizadas responden al incremento poblacional y las migraciones de personas de zonas rurales a zonas urbanas. El crecimiento acelerado de la población ha producido la ocupación de espacios disponibles, cambios en las coberturas y usos de la tierra de manera no planificada, para satisfacer las necesidades de los habitantes de las regiones consideradas.

En términos generales, los pastos con una probabilidad de $0.9866(98.7 \%)$, las áreas agrícolas heterogéneas con 0.9805 (98.1\%), los bosques con 0.9215 (92.2\%), las áreas 
Tabla 4

Transición de coberturas de uso del suelo en la macrocuenca del Pacífico durante los períodos 2000-2002 y 2005-2009

Transición 2000-2002 a 2005-2009

\begin{tabular}{lr} 
Zonas urbanizadas & 790,11 \\
\hline Zonas industriales o comerciales y redes de comunicación & 4,58 \\
\hline Zonas de extracción minera y escombreras & 322,02 \\
\hline Cultivos transitorios & $-1831,28$ \\
\hline Cultivos permanentes & 2529,73 \\
\hline Pastos & $-16142,14$ \\
\hline Áreas agrícolas heterogéneas & 530184,25 \\
\hline Bosques & $-173718,30$ \\
\hline Áreas con vegetación herbácea y/o arbustiva & 24638,53 \\
Áreas abiertas, sin o con poca vegetación & $-1112,05$ \\
\hline Áreas húmedas continentales & 5209,90 \\
Áreas húmedas costeras & $-548,44$ \\
\hline Aguas continentales & 8596,27 \\
\hline Sin información & $-378923,18$ \\
\hline
\end{tabular}

con vegetación herbáceay/o arbustiva con una probabilidad de $0.9780(97.8 \%)$, presentan las más bajas probabilidades de cambio durante este período.

Haciendo una comparación entre los dos períodos de las tendencias de cambio para estas coberturas, se puede decir que las áreas agrícolas heterogéneas ampliaron la tendencia a permanecer como cobertura importante dentro de la macrocuenca, lo que indica una consolidación en las actividades agropecuarias de los habitantes de la región como fuente de sustento a partir del uso del territorio en cultivos transitorios y como alternativa de desarrollo económico con los cultivos permanentes y las áreas de pastos, todo esto con grandes necesidades en cuanto a la demanda de recursos hídricos que favorezcan estas actividades.

Los bosques también ampliaron la tendencia a permanecer como cobertura lo que indica una disminución en la presión sobre el recurso forestal lo que de alguna manera favorece la oferta del recurso hídrico, debido al alto grado de protección que esta cobertura ejerce sobre el territorio favoreciendo así las diferentes actividades productivas y de consumo por parte de la población.

Las áreas con vegetación herbácea y/o arbustiva disminuyeron la tendencia a permanecer en esta cobertura, lo que indica que corresponden a zonas donde por una parte se han venido recuperando y que por acción natural en este momento hacen parte de coberturas boscosas, o que vienen siendo incorporadas a las diversas actividades agropecuarias que se vienen implementando en la región.
Las áreas abiertas con o sin poca vegetación, ampliaron la tendencia a permanecer en esta cobertura, y puede corresponder a zonas donde los procesos de degradación producidos por la acción antrópica han sido lo suficientemente fuertes que han dificultado su recuperación y se han convertido en territorios improductivos donde se generan fuertes impactos asociados con fenómenos de escorrentía superficial, que debido a la poca protección del suelo producen importantes procesos de erosión, los cuales inciden negativamente sobre los cuerpos de agua ocasionando graves problemas de sedimentación, repercutiendo así en la calidad y cantidad del recurso hídrico.

\section{Literatura citada}

Alcaraz D, Baldi G, Durante P, Garbulsk M. 2008. Análisis de la dinámica temporal del NDVI en áreas protegidas: tres casos de estudio a distintas escalas espaciales, temporales y de gestión. Ecosistemas. 17 (3): 108-17.

Corporación Autónoma Regional para el Desarrollo Sostenible del Chocó (CODECHOCÓ). 2015. Base de datos de especies movilizadas en 2009. Archivo digital no publicado. Quibdó: CODECHOCÓ,

Departamento Nacional de Planeación (DNP). 2007. Documento Visión Colombia 2019: Consolidar una gestión ambiental que promueva el desarrollo sostenible. [consultado mayo, 2015]. Disponible en: http:// www.dnp.gov.co/PortalWeb/Portals/0/archivos/documentos/2019/Documentos/101040-CARTILLA\%20AM- BIENTAL.pdf

Organización de Naciones Unidas para la Agricultura y laAlimentación(FAO). 2010. Evaluación de los recursos forestales mundiales 2010. Informe Nacional: Colombia. Documentos de trabajo de la evaluación de los recursos forestales mundiales. Roma: FAO.

IDEAM, IGAC, IAvH, Invemar, I. Sinchi, UTPC. 2010. Leyenda de coberturas de la Tierra CORINE Land Cober adaptada para Colombia, escala 1:100.000. Bogotá: Instituto de Hidrología, Meteorología y Estudios 


\section{Bioetnia Volumen 10, 2013}

Ambientales, Instituto Geográfico Agustín Codazzi, Instituto de Investigación de Recursos Biológicos Alexander von Humboldt, Universidad Pedagógica y Tecnológica de Colombia, Instituto de Investigaciones Marinas y Costeras José Benito Vives De Andréis e Instituto Amazónico de Investigaciones Científicas Sinchi.

Instituto de Hidrología, Meteorología y Estudios Ambientales (IDEAM).

2010. Resumen ejecutivo de la memoria técnica de la cuantificación de la deforestación histórica para Colombia. Bogotá: IDEAM. 17 pp.

Instituto de Hidrología, Meteorología y Estudios Ambientales IDEAM. 2011. Análisis de tendencias y patrones espaciales de deforestación en
Colombia. Bogotá: IDEAM.

Instituto Geográfico Agustín Codazzi (IGAC y CORPOICA). 2002. Zonificación de los conflictos de uso de la tierra en Colombia. Bogotá: IGAC y CORPOICA.

Ruiz V, Save R, Herrera A. 2013. Análisis multitemporal del cambio de uso del suelo, en el Paisaje Terrestre Protegido Miraflor Moropotente, Nicaragua 1993-2011. Ecosistemas. 22 (3): 117-23.

Organización de Naciones Unidas contra la Droga y el Delito (UNODC). 2010. Monitoreo de cultivos de coca 2009. Bogotá: UNODC. 